\title{
Hurwitz on Hadamard designs
}

\section{T. Storer}

An $n \times n$-matrix on $n$ signed variables is called Hadomard of Wizliconson type if each variable occurs exactly once in each row, and the inner product of any pair of distinct rows is zero. We show here that these matrices correspond in a natural way to rational formulas for products of sums of $n$ squares, shown by Hurwitz to exist only for $n=1,2,4$, and 8 . Hurwitz' arguments contain an implicit proof that this correspondence is one-to-one (we show this directly) and hence that Hadamard matrices of Williamson type exist for orders $1,2,4$ and 8 only.

An Hadomard design $[1,2]$ is an $n \times n$ array $H(n ; k)$ of $k$ signed variables ("letters") with the property that the inner product of any two distinct rows of the array is zero. Such a design has been said to be of Wizzionson type (after [6]) if $k=n$ and each letter occurs exactly once in each row of $H^{(n ; n) \triangleq H^{(n)}}$, and it has recently been shown [5] that $H^{(n)}$ exists if and only if $n=1,2,4$, or 8 . The purpose of this note is to show that this result was known to Hurwitz [3, 4].

If

$$
\left(x_{1}^{2}+x_{2}^{2}+\ldots+x_{n}^{2}\right)\left(y_{1}^{2}+y_{2}^{2}+\ldots+y_{n}^{2}\right)=P_{1}^{2}+P_{2}^{2}+\ldots+p_{n}^{2},
$$

where the $P_{i}$ are bilinear forms in the $x_{j}, y_{k}$, we may form an $n \times n$ matrix $H=\left[H_{i j}\right]$ whose $i j$-entry $H_{i j}$ is the (signed) coefficient of $x_{i}$ in $P_{j}$. In the special case $n=4$, for example,

Received 15 September 1970. The author was supported in part by a National Science Foundation research grant. 


$$
\begin{aligned}
& P_{1}=x_{1} y_{1}+x_{2} y_{2}+x_{3} y_{3}+x_{4} y_{4}, \quad P_{2}=x_{1} y_{2}-x_{2 y_{1}}+x_{3} y_{4}-x_{4} y_{3}, \\
& P_{3}=x_{1} y_{3}-x_{2 y_{4}-x_{3} y_{1}+x_{4} y_{2},} P_{4}=x_{1} y_{4}+x_{2} y_{3}-x_{3} y_{2}-x_{4} y_{1},
\end{aligned}
$$
and

$$
H=\left[\begin{array}{rrrr}
y_{1} & y_{2} & y_{3} & y_{4} \\
y_{2} & -y_{1} & y_{4} & -y_{3} \\
y_{3} & -y_{4} & -y_{1} & y_{2} \\
y_{4} & y_{3} & -y_{2} & y_{1}
\end{array}\right]
$$

is Hadamard of Williamson type. Hurwitz essentially showed that a formula of type (1) exists for a given $n$ if and only if $n=1,2,4$, or 8 , and that each of these formulas leads to an $H^{(n)}$ (which he exhibits in [3], p. 570) of the corresponding order. Thus, if it could be shown that to each $H^{(n)}$ there corresponds a formula of type (1), the admissible orders $n$ for $H^{(n)}$ would also be known. This is implicit in Hurwitz' discussion, being, in fact, almost trivial. For, given an Hadamard matrix $H^{(n)} \triangleq H=\left[H_{i j}\right]$, define bilinear forms $P_{1}, P_{2}, \ldots, P_{n}$ by the previous correspondence; further define $\hat{H}=\left[\hat{H}_{i j}\right]$ where, if $H_{i k}= \pm y_{j}$, then $\hat{H}_{i j}= \pm x_{k}$. Clearly $\hat{H}$ is Hadamard of Williamson type and, if

$$
x=x_{1}^{2}+x_{2}^{2}+\ldots+x_{n}^{2}, \quad y=y_{1}^{2}+y_{2}^{2}+\ldots+y_{n}^{2},
$$

then

$$
H^{T} H \hat{H} \hat{H}^{T}=\left(H \hat{H}^{T}\right)\left(H \hat{H}^{T}\right)^{T}=X Y . I \quad(I=n \times n \text { identity matrix }) .
$$

But

$$
\left(H \hat{H}^{T}\right)_{i j}=\sum_{k=1}^{n} H_{i k} \hat{H}_{j k} \not \equiv 0, \quad\left(H \hat{H}^{T}\right)_{i j}^{T}=\sum_{k=1}^{n} H_{j k} \hat{H}_{i k} \not 0
$$

and

$$
H_{i k} \hat{H}_{j k}=\text { (coeff. of } x_{k} \text { in } P_{i} \text { ). (coeff. of } y_{k} \text { in } P_{j} \text { ). }
$$

If, now, $y_{s}=$ (coeff. of $x_{k}$ in $P_{i}$ ), let 
$x_{\sigma(k)}=$ (coeff. of $y_{s}$ in $P_{j}$ ); this defines a permutation $\sigma$ of the n-set. Further, from the definition of $H$, we have (coeff. $x_{\sigma(k)}$ in $\left.P_{i}\right)= \pm$ (coeff. $x_{k}$ in $P_{j}$ ); thus $\mid\left(\operatorname{coeff}\right.$. of $x_{k}$ in $\left.P_{i}\right) \cdot\left(\operatorname{coeff}\right.$. of $y_{k}$ in $\left.P_{j}\right) \mid=$ $\mid\left(\operatorname{coeff}\right.$. of $x_{\sigma(k)}$ in $\left.P_{j}\right) \cdot\left(\operatorname{coeff}\right.$. of $y_{\sigma(k)}$ in $\left.P_{i}\right) \mid$ and hence $H=\left[\left|\left(H H^{T}\right)_{i j}\right|\right]$ is symmetric. This implies that $X Y$ is a sum of $n$ squares of bilinear forms in the $x_{i}, y_{j}$. Continuing with the case $n=4$, for example, we find

$$
H \hat{H}^{T}=\left[\begin{array}{cccc}
P_{1} & P_{2} & P_{3} & P_{4} \\
P_{2} & -P_{1} & P_{4} & -P_{3} \\
P_{3} & -P_{4} & -P_{1} & P_{2} \\
P_{4} & P_{3} & -P_{2} & -P_{1}
\end{array}\right]
$$

which is, again, Hadamard of Williamson type, as expected.

As a final interesting note, Hurwitz' closing remark in [3] implies that an Hadamard design of order 16 on $p$ letters, each of which must occur exactly once in each row, must satisfy

$$
p<\frac{2 \log 16}{\log 2}+2 \sim 10.1
$$

that is $p \leq 10$.

\section{References}

[1] L.D. Baumert and Marshall Hall, Jr, "A new construction for Hadamard matrices", Bul2. Amer. Math. Soc. 71 (1965), 169-170.

[2] Marshall Hall, Jr, Combinatorial theory (Blaisdell Publishing Co.

[Ginn and Co.], Waltham, Massachusetts; Toronto, Ontario; London; 1967 ). 
[3] Adolf Hurwitz, "Über die Komposition der quadratischen Formen von beliebig vielen Variabeln", Nachr. Königl. Ges. Wiss. Göttingen, Math.-phys. Klasse (1898), 309-316; Math. Werke, Band 2, 565-571 (Birkhäuser, Basel, 1933).

[4] A. Hurwitz, "Über die Komposition der quadratischen Formen", Math. Ann. 88 (1923), 1-25; Math. Werke, Band 2, 641-666 (Birkhäuser, Basel, 1933).

[5] Jennifer Wallis, "Hadamard matrices", BuzZ. Austral. Math. Soc. 2 (1970), 45-54.

[6] John Williamson, "Hadamard's determinant theorem and the sum of four squares", Duke J. Math. 11 (1944), 65-81.

University of Michigan,

Ann Arbor,

Michigan, USA. 